\title{
The Geometrical Phase Transitions in a Lattice Model
}

\author{
Koji Kuroda \\ Institute of Mathematics, University of Tsukuba, Sakuramura \\ Niiharigun Ibaraki 305, Japan
}

\begin{abstract}
We shall consider a finite range model on a square lattice $\mathbf{Z}^{3}$ and show the existence of bubble, tubular and lamellar phases by estimating the correlation functions at low temperature.
\end{abstract}

\section{Introduction}

In many systems such as the mixture of water and soap we can observe the phenomena of changes of the geometrical structures according to the density. When we dissolve the soap in water, the system changes from the dissipative state into the state of hexagonal structure, then into the state of lamellar structure and finally into the state of gel. The interaction in the real substances seems too complicated to be analyzed mathematically.

In this paper we shall show that such a phenomenon is realized even in the simple model, though our model may not be the simplest one. Consider the 3dimensional square lattice $\mathbf{Z}^{3}$. We arrange oil-particles and water-particles on sites of $\mathbf{Z}^{3}$, and also arrange soap-molecules on bonds of the lattice. Taking into account the orientational tendency between the hydrophobic group and the hydrophilic group of soap molecules, we set up the interactions between the "components" whose ranges are $1, \sqrt{2}$, and 2 .

We shall prove that the system changes from the state of bubble structure into the tubular structure and finally into the lamellar structure as the density of oil-particles increases by estimating the volume of disordered phase.

We state our results rigorously in Sect. 2 after preparing the necessary definitions in Sect.1. Section 3 is devoted to the proof of theorems. In this section we consider the correlation functions of Bloch walls and derive several properties of them. As our model does not have simple symmetry in the Ising model, we extend Heilmann's method [3] to obtain the upper bound on the correlation functions.

By using this estimate we obtain the unique solution of the correlation equation, and we also have several properties of correlation functions by refining the Minlos-Sinai method [1,2]. 


\section{Description of the Model}

Consider a 3-dimensional square lattice $\mathbf{Z}^{3}$. Suppose that each site of $\mathbf{Z}^{3}$ can be occupied by any of the two types of particles called oil-particles and waterparticles, and that each bond of the lattice can be occupied by a soap-molecule or nothing. We denote a soap-molecule by an arrow $(\rightarrow)$, and regard the head of the arrow as a hydrophobic group and the tail as a hydrophilic group. (See Fig. 1.) For simplicity we denote an oil-particle and a water-particle by an $o$ particle and a $w$-particle respectively.

Before describing the interactions on the system we prepare some terminologies which will be used in the sequel. Let $|x-y|$ be the Euclidean distance between $x$ and $y \in \mathbf{Z}^{3}$. We say two sites $x \in \mathbf{Z}^{3}$ and $y \in \mathbf{Z}^{3}$ are adjacent if $|x-y|$ $=1$. A subset $V$ of $Z^{3}$ is called connected if for all $x \in V$ and $y \in V(x \neq y)$ there is a path in $V x_{1}=x, x_{2}, \ldots, x_{n}=y$ such that $x_{i}$ and $x_{i+1}$ are adjacent $(i$ $=1,2, \ldots, n-1)$. For a given $V \subset \mathbf{Z}^{3}$, we denote the set of all sites of $\mathbf{Z}^{3} \backslash V$ adjacent to some sites of $V$ by $\partial V$ and the set of all sites of $V$ adjacent to some sites of $Z^{3} \backslash V$ by $\partial_{\text {in }} V$. We say that a pair of bonds is "perpendicular" if they contact at one site and are perpendicular. Let $T$ be the totality of sites and bonds in $\mathbf{Z}^{3}$. In the usual way the configuration space is defined on $T$.

Now we describe the interactions between the "components" in the following.

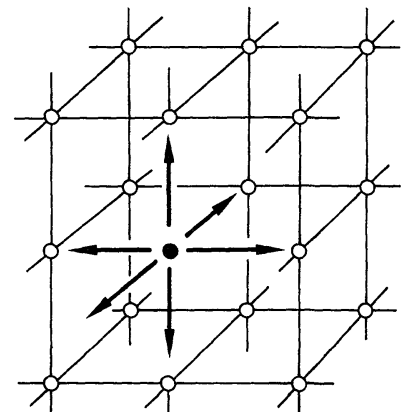

1) Bubble structure

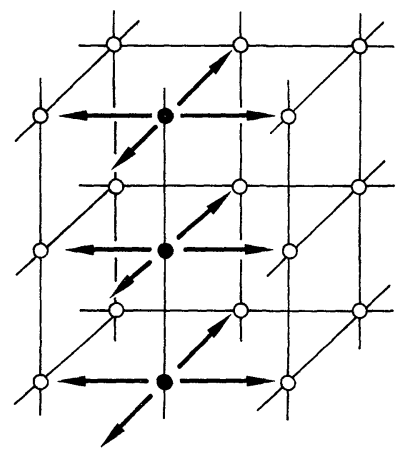

2) Tubular structure

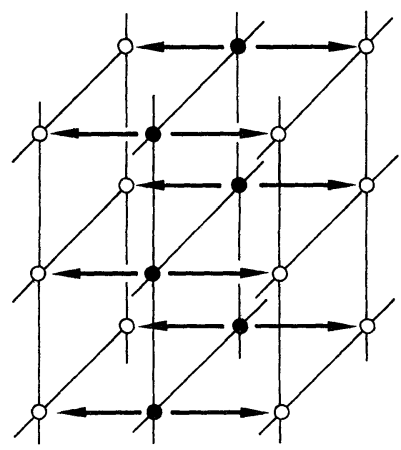

3) Lamellar structure

Fig. 1 


\begin{tabular}{l|r|r|r} 
Distance & 1 & $\sqrt{2}$ & 2 \\
\hline Attractive & $-\varepsilon_{1}$ & $-\varepsilon_{3}$ & $-\varepsilon_{5}$ \\
Repulsive & $\varepsilon_{2}$ & $\varepsilon_{4}$ & $\varepsilon_{6}$
\end{tabular}

Fig. 2
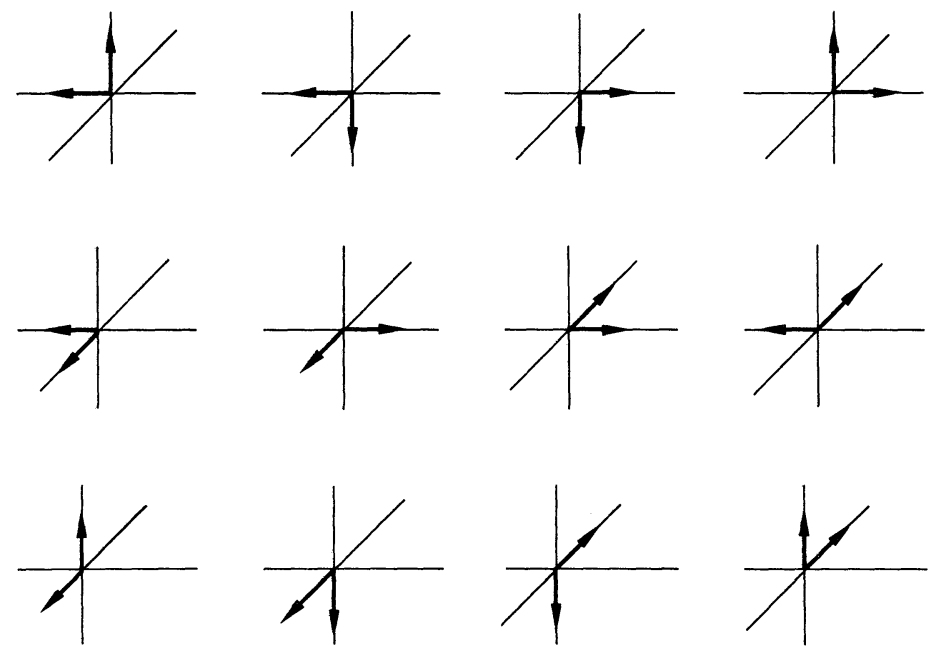

Fig. 3

1) The interaction potentials between oil-oil pairs, oil-water pairs, and water-water pairs, at a distance $\mathbf{r}$, are given by

$$
\begin{aligned}
U_{o, o}(\mathbf{r}) & \equiv 0 \\
U_{o, w}(\mathbf{r}) & =\left\{\begin{array}{ll}
\varepsilon_{0} & \text { for }|\mathbf{r}|=1 \\
0 & \text { otherwise }
\end{array}\left(\varepsilon_{0}>0\right)\right. \\
U_{w, w}(\mathbf{r}) & \equiv 0 .
\end{aligned}
$$

2) Soap-molecules interact with particles located on the nearest sites to the molecules as follows; if a hydrophobic group of a soap-molecule contacts with an $o$-particle or a hydrophilic group contacts with a $w$-particle, the interaction is repulsive and its potential energy is given by $2 \varepsilon_{0}$, while if a hydrophilic group of molecule contacts with an $o$-particle or a hydrophobic group contacts with a $w$-particle, it is attractive and its potential energy is given by $-\varepsilon_{0}$.

3-1) Two soap-molecules located on two parallel bonds interact with each other as follows; the interaction is attractive if they point in the same direction, while it is repulsive if they point in the opposite directions, and the potential energies depend on distances between them and are shown in Fig. 2.

3-2) Two soap-molecules located on a perpendicular pair of bonds interact with each other as follows; the attractive interaction with potential energy $-\varepsilon_{7}$ $\left(\varepsilon_{7}>0\right)$ is given if it is one of the 12 types of pairs shown in Fig. 3, otherwise the repulsive interaction with energy $\varepsilon_{8}\left(\varepsilon_{8}>0\right)$ is given. 
3-3) No interaction is given to the remaining pairs of soap-molecules.

4) We set the chemical potential at $8 \mu$ for an $o$-particle and zero for others.

Next we introduce the notions of the block configuration and the block energy. Let $S$ be the dual lattice of $\mathbf{Z}^{3}$. We call the totality of sites and bonds in a unit cube a "block" and represent it by its center $t \in S$. Note that $\varepsilon_{1}, \varepsilon_{2}, \varepsilon_{7}$, and $\varepsilon_{8}$-pairs are common to two nearest neighbour blocks and moleculeparticle pairs are common to four nearest neighbour blocks. We now define the total potential energy $E_{t}(\xi)$ is a block $t \in S$ under the configuration $\xi$ by

$$
\begin{aligned}
E_{t}(\xi)= & \frac{1}{2}\left(-n_{1}(\xi) \varepsilon_{1}+n_{2}(\xi) \varepsilon_{2}-n_{7}(\xi) \varepsilon_{7}+n_{8}(\xi) \varepsilon_{8}\right) \\
& -n_{3}(\xi) \varepsilon_{3}+n_{4}(\xi) \varepsilon_{4}-\mu n(\xi) \\
& +\frac{1}{4}(\text { the total energy of particle-molecule pairs in } t)
\end{aligned}
$$

where $n(\xi), n_{i}(\xi)$ are the number of $o$-particles, $\varepsilon_{i}$-pairs $(i=1,2, \ldots, 8)$ in $t \in S$.

Let $\Sigma$ be the set of all configurations in a block. Put $\Omega=\Sigma^{S}$. We say $\omega \in \Omega$ is consistent if for each nearest neighbour pairs of blocks $\left(t_{1}, t_{2}\right)$

$$
\left.\omega\left(t_{1}\right)\right|_{t_{1} \cap t_{2}}=\left.\omega\left(t_{2}\right)\right|_{t_{1} \cap t_{2}}
$$

where $\left.\omega\left(t_{i}\right)\right|_{t_{1} \cap t_{2}}$ is the restriction of the block configuration $\omega\left(t_{i}\right)$ on the common part $t_{1} \cap t_{2}$ of the blocks $t_{1}$ and $t_{2}$.

Let $\hat{\Omega}$ be the set of all consistent configurations, then there is a one-to-one correspondence between the original configuration space and $\hat{\Omega}$. For each $\xi \in \hat{\Omega}$ and each nearest neighbour pair of blocks $\left(t_{1}, t_{2}\right)$, we define the mutual potential energy $E_{t_{1}, t_{2}}(\xi)$ between $t_{1}$ and $t_{2}$ by

$$
E_{t_{1}, t_{2}}(\xi)=\frac{1}{2}\left(-\varepsilon_{5} n_{5}(\xi)+\varepsilon_{6} n_{6}(\xi)\right)
$$

where $n_{5}(\xi)$ and $n_{6}(\xi)$ are the number of $\varepsilon_{5}$ and $\varepsilon_{6}$ pairs in $t_{1} \cup t_{2}$ under $\xi$ respectively.

Further, the block energy $E(t ; \xi)$ of the block $t$ is defined by

$$
E(t ; \xi)=E_{t}(\xi(t))+\frac{1}{2} \sum_{s:|t-s|=1} E_{t, s}(\xi)
$$

For any $V \subset S$, let $\hat{\Omega}_{V}$ be the set of all consistent configurations in $V$. We say $\xi \in \hat{\Omega}_{V}$ is consistent with respect to $\omega \in \hat{\Omega}$ if $(\xi, \omega) \in \hat{\Omega}$, where $(\xi, \omega)$ is the configuration given by

$$
(\xi, \omega)(t)=\left\{\begin{aligned}
\xi(t) & \text { whenever } t \in V \\
\omega(t) & \text { otherwise. }
\end{aligned}\right.
$$

Let $\hat{\Omega}_{V, \omega}$ be the set of all configurations $\xi \in \hat{\Omega}_{V}$ consistent with $\omega \in \hat{\Omega}$.

With the notions above we define the Gibbs distribution. The Gibbs distribution in the finite set $V \subset S$, with the boundary condition $\omega \in \hat{\Omega}$ is the probability distribution

$$
P_{V . \omega}(\xi)=\frac{1}{Z_{V}(\omega)} \exp \left\{-\beta U_{V}(\xi \mid \omega)\right\} \quad\left(\xi \in \hat{\Omega}_{V . \omega}\right)
$$


where $U_{V}(\xi \mid \omega)$ and $Z_{V}(\omega)$ are given by

$$
\begin{aligned}
U_{V}(\xi \mid \omega) & =\sum_{t \in V \cup \partial V} E(t ;(\xi, \omega)), \\
Z_{V}(\omega) & =\sum_{\xi \in \hat{\Omega}_{V}, \omega} \exp \left\{-\beta U_{V}(\xi \mid \omega)\right\} .
\end{aligned}
$$

A random field $\left\{X_{t} ; t \in S\right\}$ on $\hat{\Omega}$ is called a Gibbs random field if for every finite $V \subset S$ its conditional distributions are given by

$$
\operatorname{Pr}\left\{X_{t}=\xi(t), t \in V \mid X_{t}=\omega(t), t \in S \backslash V\right\}=P_{V, \omega}(\xi)
$$

for all $\xi \in \hat{\Omega}_{V, \omega}$ and almost all $\omega \in \hat{\Omega}$.

\section{The Statements of the Results}

In this section we state our results. We shall show that the system changes from the state of the bubble structure into the tubular structure, and finally into the lamellar structure as the value of $\mu$ increases. Also we shall consider the asymptotic properties of the canonical Gibbs measures in the limit as $V \rightarrow \mathbf{Z}^{3}, N \rightarrow \infty, \frac{N}{V} \rightarrow n^{*}$, where $n^{*}$ is the density of $o$-particles.

To begin with we consider for which configurations the block energy in each block takes minimal value. Assume that the interaction parameters $\varepsilon_{0}, \varepsilon_{2}$, and $\varepsilon_{4}$ are sufficiently large compared with other interaction parameters, then $E_{t}(\xi)(\xi \in \Sigma)$ can take minimal value only at the following ten types of configurations in $\Sigma$. (See Fig. 4.)

Let $\Sigma_{i}$ be the set of all $A_{i}$-type configurations in $\Sigma(i=0,1, \ldots, 9)$. We catalog the numbers of elements in these sets:

$$
\begin{aligned}
& \#\left(\Sigma_{0}\right)=\#\left(\Sigma_{9}\right)=1, \quad \#\left(\Sigma_{1}\right)=\#\left(\Sigma_{2}\right)=\#\left(\Sigma_{5}\right)=\#\left(\Sigma_{6}\right)=\#\left(\Sigma_{8}\right)=8 \\
& \#\left(\Sigma_{2}\right)=\#\left(\Sigma_{7}\right)=12, \quad \text { and } \quad \#\left(\Sigma_{4}\right)=6 .
\end{aligned}
$$

Let $\Pi_{i}$ be the set of all configurations $\omega$ in $\hat{\Omega}$ such that $\omega(t) \in \Sigma_{i}$ for each $t \in S$. Clearly the number of elements in $\Sigma_{i}$ is equal to the number of elements in $\Pi_{i}$. It is easily seen that each element of $\Pi_{1}, \Pi_{2}$, and $\Pi_{4}$ expresses, geometrically, the bubble structure, the tubular structure, and the lamellar structure respectively. We also note that the block energy $E(t ; \omega)$ is independent of $t \in S$ whenever $\omega \in \Pi_{i}(i=0,1, \ldots, 9)$.

Under the following three conditions $\mathrm{c}-1, \mathrm{c}-2$, and $\mathrm{c}-3$

c-1) $3 \varepsilon_{8}+4 \varepsilon_{1}>3 \varepsilon_{7}+2 \varepsilon_{3}+2 \varepsilon_{5}$

c-2) $\varepsilon_{1}+\varepsilon_{5}>2 \varepsilon_{3}$

c-3) $\operatorname{Min}\left(\varepsilon_{1}+\varepsilon_{5}+\varepsilon_{8}, 2 \varepsilon_{1}+3 \varepsilon_{3}+\varepsilon_{5}\right)>\varepsilon_{7}>2 \varepsilon_{1}+\varepsilon_{5}$,

the following assertions are obtained; 

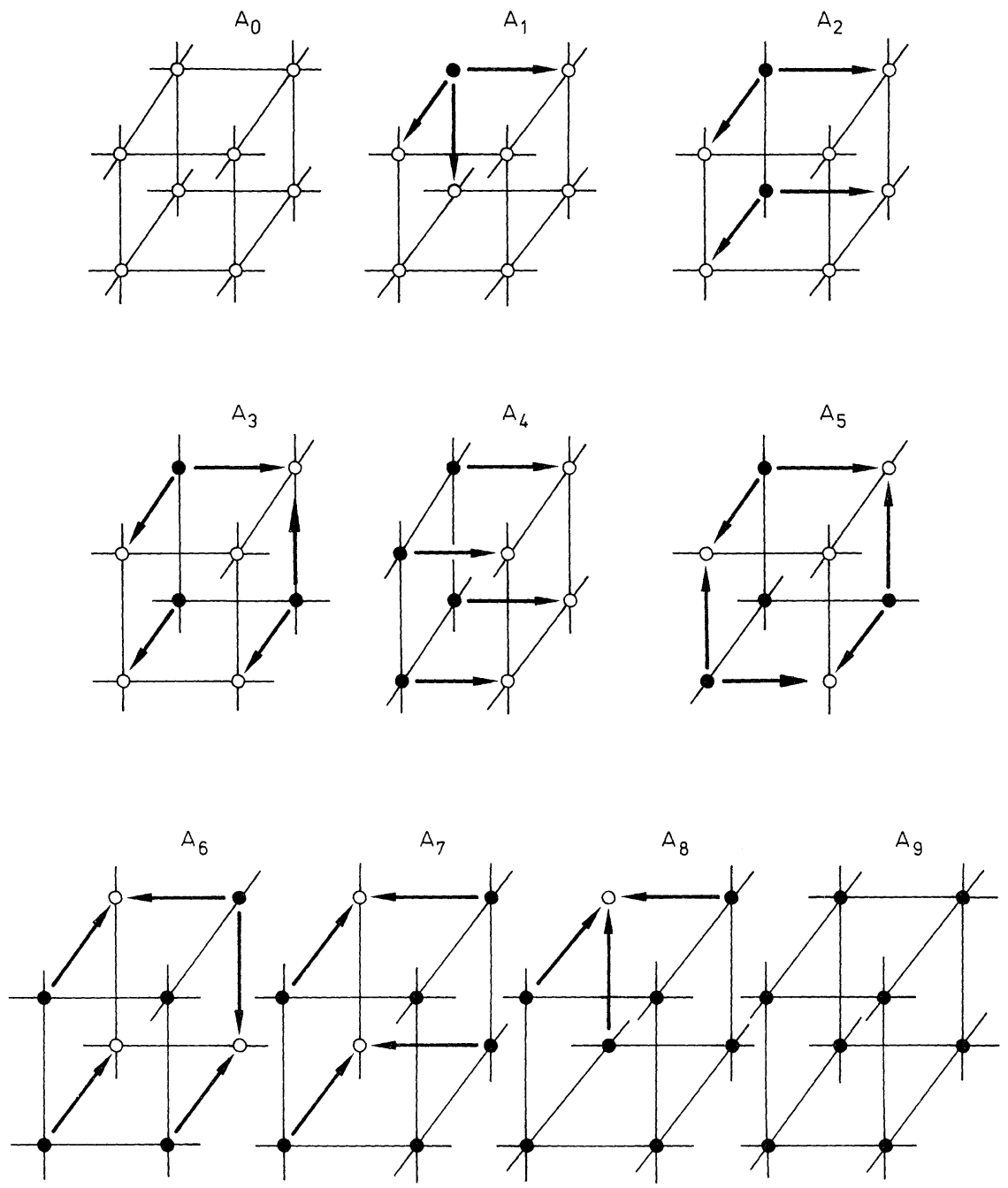

Fig. 4

1) if $\mu \in K_{1}=\left[0, \mu_{1}\right)$, then $E\left(t ; \omega_{1}\right)<E(t ; \omega)$ for all $\omega_{1} \in \Pi_{1}$ and all $\omega \in \widehat{\Omega} \backslash \Pi_{1}$,

2) if $\mu \in K_{2}=\left(\mu_{1}, \mu_{2}\right)$, then $E\left(t ; \omega_{2}\right)<E(t ; \omega)$ for all $\omega_{2} \in \Pi_{2}$ and all $\omega \in \widehat{\Omega} \backslash \Pi_{2}$,

3) if $\mu \in K_{4}=\left(\mu_{2}, \mu_{3}\right)$, then $E\left(t ; \omega_{4}\right)<E(t ; \omega)$ for all $\omega_{4} \in \Pi_{4}$ and all $\omega \in \widehat{\Omega} \backslash \Pi_{4}$,

where $\mu_{1}=\frac{1}{2} \varepsilon_{7}-\varepsilon_{1}-\frac{1}{2} \varepsilon_{5}, \mu_{2}=\frac{1}{2} \varepsilon_{7}-\frac{1}{2} \varepsilon_{1}-\varepsilon_{3}$, and $\mu_{3}=\frac{1}{2} \varepsilon_{1}+\varepsilon_{3}+\frac{1}{2} \varepsilon_{8}$.

Put $\Pi_{i}=\left\{\omega_{1}^{(i)}, \omega_{2}^{(i)}, \ldots, \omega_{N_{2}}^{(i)}\right\}(i=1,2,4)$, where $N_{1}=8, N_{2}=12$, and $N_{4}=8$. By using the assertions above and the Heilmann's argument, we can prove the occurrence of phase transitions. 
Theorem 1. For any fixed $\mu \in K_{i}(i=1,2,4)$ and any finite subset $C \subset S$, there exists a function $g_{i}(\beta)$, tending to zero as $\beta$ tends to infinity, and at least $N_{i}$ distinct limiting Gibbs measures $P_{\omega_{J}^{(i)}}\left(j=1,2, \ldots, N_{i}\right)$ which satisfy the following estimates,

$$
P_{\omega_{j}^{(\nu)}}\left(X_{t}=\omega_{j}^{(i)}(t) ; t \in C\right) \geqq 1-g_{i}(\beta) \quad\left(j=1, \ldots, N_{i}\right)
$$

for sufficiently large $\beta$.

To describe the next result we introduce the following definitions. We fix a finite cube $V \subset S$ and a boundary condition $\omega \in \Pi_{1} \cup \Pi_{2} \cup \Pi_{4}$. For any $\xi \in \hat{\Omega}_{V \text {, } \omega}$, the block $t \in V$ is called static if $\xi(s)=\omega(s)$ for all $s$ satisfying $|s-t| \leqq 1$, and is called active if it is not. For any $\omega \in \Pi_{i}$ and any $\mu \in K_{i}, E(t ; \xi)$ is the minimal value if $t$ is static under $\xi \in \hat{\Omega}_{V, \omega}$. The totality of active sites is denoted by $\mathbf{B}(\xi)$. For any $\xi \in \hat{\Omega}_{V, \omega}$ there is a unique decomposition of $\mathbf{B}(\xi)$ into the connected components $\left\{B_{1}(\xi), \ldots, B_{s}(\xi)\right\}$. A couple $\bar{B}_{i}=\left(B_{i},\left(\xi(t) ; t \in B_{i}\right)\right)$ of the connected component $B_{i}$ and the configuration on it is called $B$-wall (Bloch wall). We say $\bar{B}$ is an outer $B$-wall if it is contained in no other $B$-walls. We call the region enclosed by the outer boundaries of outer $B$-walls "the disordered phase", and denote it by $D(\xi)$. On the other hand the region $O(\xi)=V \backslash D(\xi)$ is called "the ordered phase". We say $\bar{B}_{1}=\left(B_{1},\left(\xi(t) ; t \in B_{1}\right)\right)$ is congruent to $\bar{B}_{2}=\left(B_{2},(\zeta(t)\right.$; $\left.t \in B_{2}\right)$ ), if $B_{1}$ is superimposed on $B_{2}$ by the translation $T$ and $\xi(t)=\zeta(T(t))$ for all $t \in B_{1}$. A congruence class of $B$-wall is denoted by $\bar{\gamma}$, and the set of such congruence classes is denoted by $\Gamma$. Also the volume of the region enclosed by the outer boundary of $\gamma$ is denoted by $v(\gamma)$.

Put

$$
\delta^{*}(\beta, \mu)=\sum_{\bar{\gamma} \in \Gamma} v(\gamma) \rho(\bar{\gamma} ; \beta, \mu)
$$

where $\rho(\bar{\gamma} ; \beta, \mu)$ is the limiting correlation function of $\bar{\gamma} \in \Gamma$ and is defined in the next section.

Let $N_{\text {oil }}(\xi)$ be the number of oil-particles in $V$ under the configuration $\xi \in \widehat{\Omega}_{V, \omega}$. For each $j \in\{1,2,4\}$, put

$$
n_{j}^{* *}=u_{j}-\sum_{\bar{\gamma} \in \Gamma}\left(u_{j} v(\gamma)-\langle n(\bar{\gamma})\rangle\right) \rho(\bar{\gamma} ; \beta, \mu),
$$

where $u_{1}=\frac{1}{8}, u_{2}=\frac{1}{4}, u_{4}=\frac{1}{2}$, and $\langle n(\bar{\gamma})\rangle$ is the expectation value of $N_{\text {oil }}$ in the Gibbs ensemble $\Upsilon(\bar{\gamma} ; \beta, \mu)$ whose exact definition is given in the next section. From the estimates of correlation functions given in the next section, we have $n_{j}^{* *}(\beta, \mu) \rightarrow u_{j}$ as $\beta \rightarrow \infty$.

For any function $f(\xi)$ on $\Omega_{V, \omega}$ we denote the expectation value and the variance of $f(\xi)$ with respect to the probability measure $P_{V, \omega}$ by $\langle f\rangle_{V, \omega}$ and $V_{V, \omega}(f)$ respectively.

As for the expectation values and variances of $N_{\text {oil }}(\xi)$ and $D(\xi)$, the following estimates are obtained.

Proposition 1. For any $\omega \in \Pi_{i}, \mu \in K_{i}$, and every sufficiently large $\beta$, the following assertions 1)-4) are satisfied.

1) $\left|\left\langle N_{\mathrm{oi} 1}\right\rangle_{V, \omega, \mu}-n_{i}^{* *}\right| V||<F_{1}(\beta)|V|^{\frac{1}{2}}, F_{1}(\beta) \downarrow 0$ exponentially as $\beta \rightarrow \infty$.

2) $\left|\langle D\rangle_{V, \omega, \mu}-\delta^{*}(\beta, \mu)\right| V||<F_{2}(\beta)|V|^{\frac{1}{2}}, F_{2}(\beta) \downarrow 0$ exponentially as $\beta \rightarrow \infty$. 
3) $V_{V, \omega, \mu}\left(N_{\text {oil }}\right)<F_{3}(\beta)|V|, F_{3}(\beta) \downarrow 0$ exponentially as $\beta \rightarrow \infty$,

4) $V_{V, \omega, \mu}(D)<F_{4}(\beta)|V|, F_{4}(\beta) \downarrow 0$ exponentially as $\beta \rightarrow \infty$.

We define the canonical Gibbs measure $P_{V, \omega}^{N}(\cdot)$ by

$$
P_{V, \omega}^{N}(\cdot)=P_{V, \omega}\left(\cdot \mid N_{\text {oil }}(\xi)=N\right)
$$

Note that $P_{V, \omega}^{N}$ is independent of $\mu$.

Our main results are included in the following two theorems.

Theorem 2. For any $\mu \in K_{i}$ and any $\omega \in \Pi_{i}(i=1,2,4)$ there exist functions $f(\beta)$ and $h(\beta)$, both of which tend to zero as $\beta$ tends to infinity and satisfy

$$
\begin{array}{r}
P_{V, \omega}\left(|| D(\xi)\left|-\delta^{*}\right| V||>f(\beta)|V|^{\frac{1}{2}}\right)<h(\beta) \\
P_{V, \omega}\left(\left|N_{\text {oil }}(\xi)-n_{i}^{* *}\right| V||>f(\beta)|V|^{\frac{1}{2}}\right)<h(\beta)
\end{array}
$$

for sufficiently large $\beta$.

Theorem 3. For any $\omega \in \Pi_{i}(i=1,2,4)$ and every sufficiently large $\beta$, there exists a function $g(\beta)$ tending to zero as $\beta \rightarrow \infty$, and satisfying the following; for each $\alpha$ and each $N$ satisfying

$$
0<\alpha<\frac{1}{4} \quad \text { and } \quad\left|N-n_{i}^{* *}(\beta, \mu)\right| V||<F_{1}(\beta)|V|^{\frac{1}{2}} \quad\left(\mu \in K_{i}\right)
$$

we have

$$
P_{V, \omega}^{N}\left(|| D(\xi)\left|-\delta^{*}\right| V||>g(\beta)|V|^{\frac{3}{4}+\alpha}\right)<C(\beta) \frac{1}{|V|^{2 \alpha}},
$$

where $C(\beta)$ is some constant.

\section{Correlation Functions of $\boldsymbol{B}$-walls}

In this section we define the correlation function and derive the several properties. For a finite cube $V \subset S$ and a boundary condition $\omega \in \hat{\Omega}$, let $\tau_{V, \omega}(\bar{B})$ be the probability that $\bar{B}$ is contained in some configuration as a $B$-wall. Let $\Delta_{V, \omega}(\bar{B})$ be the set of all configurations which contains $\bar{B}$ as a $B$-wall, then $\tau_{V, \omega}(\bar{B})$ is expressed in the following form,

$$
\tau_{V, \omega}(\bar{B})=\frac{1}{Z_{V}(\omega)} \sum_{\xi \in \Delta_{V, \omega}(\bar{B})} \exp \left\{-\beta U_{V}(\xi: \omega)\right\}
$$

We define the energy of $B$-wall $\bar{B}$ by

$$
E(\bar{B})=\sum_{t \in B} E(t ; \xi) \quad\left(\xi \in \Delta_{V, \omega}(\bar{B})\right) .
$$

Note that $E(\bar{B})$ is independent of $\xi \in \Delta_{V, \omega}(B)$.

We modify the definition of $E(t ; \xi)$ so that the minimal value is zero by subtracting the minimal value. With the modification above the definition of 
$P_{V, \omega}(\cdot)$ does not change. We can express $\tau_{V, \omega}(\bar{B})$ in the following form,

$$
\tau_{V, \omega}(\bar{B})=\frac{1}{Z_{V}(\omega)} \sum_{\xi \in \Delta_{V, \omega}(\bar{B})} \prod_{\bar{B} \in \mathbf{B}(\xi)} \exp \{-\beta E(\bar{B})\},
$$

where $\mathbf{B}(\xi)$ is the set of all $B$-walls under $\xi$.

To prove the Theorem 1 we shall show the estimate,

$$
\tau_{V, \omega}(\bar{B})<\exp \left(-c_{1} \beta|B|\right),
$$

where $c_{1}$ is the absolute constant.

If the estimate (3.4) is obtained, Theorem 1 will follow by the standard argument that if the configuration of a block $t$ is not given by $\omega(t)$ it must be inside some $B$-wall.

For any $\mu \in K_{i}$ and a given configuration $\xi \in \hat{\Omega}_{V, \omega}$, we take one $B$-wall $\bar{B}(\xi)$. We say $\bar{B}(\xi)$ is $\omega_{k}^{(i)}$-type if $\xi(t)=\omega_{k}^{(i)}(t)$ for all elements in the outer boundary of $B$. (Note that each block in the outer boundary of $B$ is static, so that the configuration in the outer boundary of $B$ is given by some element in $\Pi_{i}$.)

In case of $\mu \in K_{1}$, there exists a lattice translation $T_{i, j}$ which transforms any $\omega_{i}^{(1)}$-type $B$-wall $\bar{B}$ into any other $\omega_{j}^{(1)}$-type $B$-wall. As the energy of $B$-wall is invariant under the translation, we can easily obtain the estimate (3.4) by employing the Peierl's argument.

On the other hand, in case of $\mu \in K_{2} \cup K_{4}$, we must use the Heilmann's method [3] in order to obtain the estimate (3.4). By using the reflection transformation, Heilmann has obtained the upper bound on the probability of a given contour in the models, where one has more than two structures, each of which can be transformed into any other structures by a reflection. To extend Heilmann's method to our model we have only to notice the following three facts 1)-3).

1) Each $\omega_{i}^{(j)}$-type $B$-wall $(j=2$ or 4$)$ can be transformed into any of the other types $B$-walls by the reflection or composition of reflections.

2) There exists a lower bound on the energy of a $B$-wall which is proportional to the number of elements of a $B$-wall.

3) The energy of $B$-wall is invariant under the reflection.

Next we introduce the correlation functions of outer $B$-walls and the correlation equations.

Let $V$ be the finite subset of $S$. We denote the region enclosed by the outer boundary of $V$ by $\theta(V)$, and call the set $\operatorname{In}(V)=\theta(V) \backslash V$ inner region of $V$. For any $\mu \in K_{i}$ and any $\omega \in \Pi_{i}$, put $\gamma_{V, \omega}=\left\{\mathbf{B}=\left(\bar{B}_{1}, \ldots, \bar{B}_{s}\right)\right.$; a family of $B$-walls in $V$ which doesn't enclose the inner region of $V\}$ and $\Upsilon_{V, \omega}^{\text {out }}=\left\{\mathbf{B}=\left(\bar{B}_{1}, \ldots, \bar{B}_{s}\right)\right.$; a family of outer $B$-walls in $V$ which doesn't enclose the inner region of $V$ \}. It is easily seen that each outer $B$-wall in $B \in \Upsilon_{V, \omega}$ or $Y_{V, \omega}^{\text {out }}$ is $\omega$-type.

For a given $B$-wall $\bar{B}$, the inner region of $\bar{B}$ is uniquely decomposed into connected components $\left\{I_{1}(\bar{B}), \ldots, I_{n(B)}(\bar{B})\right\}$.

Put $J_{k}(\bar{B})=I_{k}(\bar{B}) \backslash \partial_{\text {in }} I_{k}(\bar{B})$. As the configuration in $\partial B$ is uniquely determined by the configuration in $B$, the configuration in each $\partial J_{k}(\bar{B})=\partial_{\text {in }} I_{k}(\bar{B})$ is uniquely determined by $\bar{B}$ and is denoted by $\omega_{k}(\bar{B}) \in \Pi_{i}$. We introduce the 
following probability measure on $Y_{V, \omega}$,

where

$$
P_{V, \omega}(\mathbf{B})=\frac{1}{Z(V, \omega)} \prod_{i=1}^{s} \exp \left(-\beta E\left(\bar{B}_{i}\right)\right) \prod_{k=1}^{n\left(\bar{B}_{i}\right)} Z\left(J_{k}\left(\bar{B}_{i}\right) ; \omega_{k}\left(\bar{B}_{i}\right)\right)
$$

$$
Z\left(J_{k}(\bar{B}) ; \omega_{k}(\bar{B})\right)=\sum_{\mathbf{B} \in Y_{J_{k}(\bar{B}), \omega_{k}(\bar{B})}} \prod_{\bar{B} \in \mathbf{B}} \exp (-\beta E(\bar{B})) .
$$

Now we define the correlation function of outer $B$-walls as follows,

$$
\rho_{V, \omega}\left(\bar{B}_{1}, \ldots, \bar{B}_{s}\right)=\sum_{\mathbf{B} ; \mathbf{B} \subset\left\{\bar{B}_{1}, \ldots, \bar{B}_{s}\right\}} P_{V, \omega}(\mathbf{B}) .
$$

As we proved the estimate (3.4) we can prove the following lemma.

\section{Lemma 3.1.}

$$
\rho_{V, \omega}\left(\bar{B}_{1}, \ldots, \bar{B}_{s}\right)<\prod_{i=1}^{s} \exp \left(-\beta c_{1}\left|B_{i}\right|\right)
$$

for sufficiently large $\beta$, where $c_{1}$ is an absolute constant.

These correlation functions are related by a chain of equations. By the similar way as Minlos and Sinai have derived the correlation equations in Ising model [2], we have

$$
\begin{aligned}
\rho_{V, \omega}\left(\bar{B}_{1}, \ldots, \bar{B}_{s}\right)= & \exp \left(-E\left(\bar{B}_{1}\right)\right)\left\{\rho_{V, \omega}\left(\bar{B}_{2}, \ldots, \bar{B}_{s}\right)\right. \\
& +\sum_{k=1}^{\infty} \frac{(-1)^{k}}{k !} \Sigma_{V, k}^{(1)} \rho_{V, \omega}\left(\bar{B}_{2}, \ldots, \bar{B}_{s}, \bar{F}_{i_{1}}, \ldots, \bar{F}_{i_{k}}\right) \\
& \left.-\Sigma_{V}^{(2)} \rho_{V, \omega}\left(\bar{B}_{2}, \ldots, \bar{B}_{s}, \bar{H}\right)\right\} \quad \text { if } s>1, \\
\rho_{V, \omega}\left(\bar{B}_{1}\right)= & \exp \left(-E\left(\bar{B}_{1}\right)\right) \\
& \cdot\left\{1+\sum_{k=1}^{\infty} \frac{(-1)^{k}}{k !} \Sigma_{V, k}^{(1)} \rho_{V, \omega}\left(F_{i_{1}}, \ldots, F_{i_{k}}\right)-\Sigma_{V}^{(2)} \rho_{V, \omega}(\bar{H})\right\} \quad s=1
\end{aligned}
$$

where the sum $\Sigma_{V, k}^{(1)}$ extends over all $k$-ordered pairs $\left(\bar{F}_{i_{1}}, \ldots, \bar{F}_{i_{k}}\right)$ in $Y_{V, \omega}^{\text {out }}$ such that each $F_{i_{m}}$ intersects or touches $B_{1}$, and the sum $\Sigma_{V}^{(2)}$ extends over all elements $\bar{H}$ in $Y_{V, \omega}^{\text {out }}$ which contain $B_{1}$ within its interior region.

Let $N_{k}$ be the set of all $k$-ordered pairs of outer $B$-walls in $\mathbf{Z}^{3}$. Put

$$
\mathbf{X}=\left\{\Phi=\left(\phi_{k}\right)_{k \geqq 1} ;\|\Phi\|<\infty\right\}
$$

where $\phi_{k}: N_{k} \rightarrow \mathbf{R}$ and

$$
\|\Phi\|=\sup _{k \geqq 1}\left[\sup _{\left(\bar{B}_{1}, \ldots, \bar{B}_{k}\right) \in N_{k}}\left|\phi_{k}\left(\bar{B}_{1}, \ldots, \bar{B}_{k}\right)\right| \prod_{i=1}^{k} 2^{-\left|B_{i}\right|} \exp \left(c_{1} \beta\left|B_{i}\right|\right)\right] .
$$

Then $\mathbf{X}$ becomes a Banach space. We express the correlation equations in $\mathbf{Z}^{3}$ in the equation on $\mathbf{X}$. 
Taking into account (3.5), we define the linear operator $A$ as follows,

$$
\begin{aligned}
(A \Phi)_{s}\left(\bar{B}_{1}, \ldots, \bar{B}_{s}\right)= & \exp \left(-\beta E\left(\bar{B}_{1}\right)\right)\left\{\phi_{s-1}\left(\bar{B}_{2}, \ldots, \bar{B}_{s}\right)\right. \\
& +\sum_{k=1}^{\infty} \frac{(-1)^{k}}{k !} \Sigma_{\mathbf{Z}^{3}, k}^{(1)} \phi_{s-1+k}\left(\bar{B}_{2}, \ldots, \bar{B}_{s}, \bar{F}_{i_{1}}, \ldots, \bar{F}_{i_{k}}\right) \\
& \left.-\Sigma_{\mathbf{Z}^{3}}^{(2)} \phi_{s}\left(\bar{B}_{2}, \ldots, \bar{B}_{s}, \bar{H}\right)\right\},
\end{aligned}
$$

where $\Sigma_{\mathbf{Z}^{3}, k}^{(1)}$ and $\Sigma_{\mathbf{Z}^{3}}^{(2)}$ are defined in the similar way as in (3.5) (for $s=1$ we set $\left.\phi_{0}=0\right)$.

Then the correlation equations in $\mathbf{Z}^{3}$ are expressed in the following form

$$
\rho_{\omega} \dot{=} A \rho_{\omega}+\Lambda,
$$

where $\Lambda \in \mathbf{X}$ is given by

$$
\Lambda\left(\bar{B}_{1}, \ldots, \bar{B}_{s}\right)= \begin{cases}\exp \left(-\beta E\left(\bar{B}_{1}\right)\right) & \text { if } s=1 \\ 0 & \text { otherwise. }\end{cases}
$$

We shall derive the several properties of correlation functions in $V$ and $\mathbf{Z}^{3}$ in the following. Let $\chi_{V, \omega}$ be the linear operator given by

where

$$
\left(\chi_{V, \omega} \Phi\right)_{s}\left(\bar{B}_{1}, \ldots, \bar{B}_{s}\right)=\chi_{V, \omega}\left(\bar{B}_{1}\right) \ldots \chi_{V, \omega}\left(\bar{B}_{s}\right) \phi_{s}\left(\bar{B}_{1}, \ldots, \bar{B}_{s}\right),
$$

$$
\chi_{V, \omega}(\bar{B})= \begin{cases}1 & \text { if } B_{1} \subset V \text { and } \bar{B}_{1} \text { is } \omega \text {-type } \\ 0 & \text { otherwise. }\end{cases}
$$

Then $\chi_{V, \omega} \rho_{V, \omega} \in \mathbf{X}$ and (3.5) becomes

$$
\rho_{V, \omega}=\chi_{V, \omega} \Lambda+\chi_{V, \omega} A \chi_{V, \omega} \rho_{V, \omega} .
$$

Taking into account the following two facts 1) and 2), we obtain the following lemma.

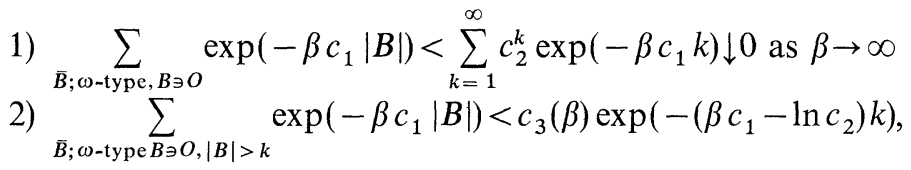
where $c_{3}(\beta) \sim 1$ as $\beta \rightarrow \infty$ and $c_{2}$ is an absolute constant.

Lemma 3.2. $\|A\|<1$ for sufficiently large $\beta$.

From this lemma Equation (3.7) has a unique solution. We call this solution a limiting correlation function. Concerning the limiting correlation function and the correlation function in $V$, we can derive several properties similar to the way Minlos and Sinai derived them for the Ising model [2].

The following two lemmas are used to derive estimates for the expectation value and the variance of the volume of the disordered physe and the number of $O$-particles. 
Lemma 3.3. If each of $\left\{\bar{B}_{1}, \ldots, \bar{B}_{s}\right\}$ is $\omega$-type and is included in $V$, then

$$
\begin{aligned}
& \left|\rho_{V, \omega}\left(\bar{B}_{1}, \ldots, \bar{B}_{s}\right)-\rho_{\omega}\left(\bar{B}_{1}, \ldots, \bar{B}_{s}\right)\right| \\
& \quad<c_{4}(\beta)\left(2 \exp \left(-c_{1} \beta\right)\right)^{\left|B_{1}\right|+\cdots+\left|B_{s}\right|} \exp \left(-\left(\beta c_{1}-\ln c_{2}\right) d\left(B_{1}, \ldots, B_{s} ; \partial V\right)\right)
\end{aligned}
$$

for sufficiently large $\beta$, where $\rho_{\omega}(\cdot)$ is the limiting correlation function and $d\left(B_{1}, \ldots, B_{s} ; \partial V\right)$ is the distance between $B_{1} \cup \ldots \cup B_{s}$ and $\partial V$.

Lemma 3.4 (Clustering Property). If both $\bar{B}_{1}$ and $\bar{B}_{2}$ are $\omega$-type and are included in $V$, then

$$
\begin{aligned}
& \left|\rho_{V, \omega}\left(\bar{B}_{1}, \bar{B}_{2}\right)-\rho_{V, \omega}\left(\bar{B}_{1}\right) \rho_{V, \omega}\left(\bar{B}_{2}\right)\right| \\
& \quad<c_{5}(\beta)\left(2 \exp \left(-c_{1} \beta\right)\right)^{\left|B_{1}\right|+\left|B_{2}\right|} \exp \left\{-\left(\beta c_{1}-\ln c_{2}\right) d\left(B_{1}, B_{2}\right)\right\}
\end{aligned}
$$

for sufficiently large $\beta$, where $c_{5}(\beta)$ is the constant depending on $\beta$.

The proof of these lemmas are given in Appendix A.

Let $\Gamma_{\omega}$ be the set of all $\omega$-type congruence classes. The expectation value and the variance of $|D(\xi)|$ and $N_{\text {oil }}(\xi)$ are given in the following forms by the standard arguement.

$$
\begin{aligned}
\langle|D(\xi)|\rangle_{V, \omega}= & \sum_{\bar{\gamma} \in \Gamma_{\omega}} v(\gamma) \sum_{\substack{\bar{B} \in \bar{\gamma} \\
B \subset V}} \rho_{V, \omega}(\bar{B}) \\
V_{V, \omega}(|D(\xi)|)= & \sum_{\substack{\bar{\gamma}_{1}, \bar{\gamma}_{2} \in \Gamma_{\omega} \\
\gamma_{1} \neq \bar{\gamma}_{2}}} v\left(\gamma_{1}\right) v\left(\gamma_{2}\right) \sum_{\substack{\bar{B}_{1} \in \bar{\gamma}_{1} B_{1} \subset V \\
\bar{B}_{2} \in \bar{\gamma}_{2} B_{2} \subset V}}\left\{\rho_{V, \omega}\left(\bar{B}_{1}, \bar{B}_{2}\right)-\rho_{V, \omega}\left(\bar{B}_{1}\right) \rho_{V, \omega}\left(\bar{B}_{2}\right)\right\} \\
& +\sum_{\bar{\gamma} \in \Gamma_{\omega}} v(\gamma)^{2} \sum_{\substack{\bar{B} \in \bar{\gamma} \\
B \subset V}} \rho_{V, \omega}(\bar{B})\left(1-\rho_{V, \omega}(\bar{B})\right) \\
\left\langle N_{\mathrm{oil}}(\xi)\right\rangle_{V, \omega}= & u_{j}|V|-\sum_{\bar{\gamma} \in \Gamma_{\omega}}\left(u_{j} v(\gamma)-\langle n(\gamma)\rangle\right) \sum_{\bar{B} \in \bar{\gamma} B \subset V} \rho_{V, \omega}(\bar{B})
\end{aligned}
$$

if $\omega \in \Pi_{j}$,

$$
\begin{aligned}
& V_{V, \omega}\left(N_{\text {oil }}(\xi)\right)=\sum_{\substack{\bar{\gamma}_{1} \bar{\gamma}_{2} \in \Gamma_{\omega} \\
\bar{\gamma}_{1} \neq \bar{\gamma}_{2}}}\left(u_{j} v\left(\gamma_{1}\right)-\left\langle n\left(\bar{\gamma}_{1}\right)\right\rangle\right)\left(u_{j} v\left(\gamma_{2}\right)-\left\langle n\left(\bar{\gamma}_{2}\right)\right\rangle\right) \\
& \cdot \sum_{\substack{\bar{B}_{1} \in \bar{\gamma}_{1} B_{1} \subset V \\
\bar{B}_{2} \in \bar{\gamma}_{2} B_{2} \subset V}}\left\{\rho_{V, \omega}\left(\bar{B}_{1}, \bar{B}_{2}\right)-\rho_{V, \omega}\left(\bar{B}_{1}\right) \rho_{V, \omega}\left(\bar{B}_{2}\right)\right\} \\
& +\sum_{\bar{\gamma} \in \Gamma_{\omega}}\left(u_{j} v(\gamma)-\langle n(\bar{\gamma})\rangle\right)^{2} \sum_{\substack{\bar{B} \in \bar{\gamma} \\
\bar{B} \subset V}} \rho_{V, \omega}(\bar{B})\left(1-\rho_{V, \omega}(\bar{B})\right)+\sum_{\bar{\gamma} \in \Gamma_{\omega}} V_{\bar{\gamma}}\left(N_{\text {oil }}\right) \sum_{\bar{B} \in \bar{\gamma}} \rho_{V, \omega}(\bar{B}),
\end{aligned}
$$

if $\omega \in \Pi_{j}$, where $\langle n(\gamma)\rangle$ and $V_{\bar{\gamma}}\left(N_{\text {oil }}\right)$ are the expectation value and variance of the number of $o$-particles in the ensemble $\Upsilon(\bar{\gamma}, \beta, \mu)$. By using Lemmas 3.3 and 3.4 Proposition 1 is easily proved.

Proof of Theorem 2. Let $m(\beta)$ be the function satisfying the following 1) and 2),

1) $m(\beta) \downarrow 0$ as $\beta \rightarrow \infty$

2) $F_{4}(\beta) / m^{2}(\beta) \downarrow 0$ as $\beta \rightarrow \infty$,

where $F_{4}(\beta)$ is the function given in Proposition 1. Put $f(\beta)=F_{2}(\beta)+m(\beta)$. 
From Proposition 1 and the Chebyshev's inequality, we have

$$
\begin{aligned}
\left.P_{V, \omega}\left(|| D(\xi)\left|-\delta^{*}\right| V||\right\rangle f(\beta)|V|^{\frac{1}{2}}\right) & <P_{V, \omega}\left(|| D(\xi)\left|-\langle|D(\xi)|\rangle_{V, \omega}\right|>m(\beta)|V|^{\frac{1}{2}}\right) \\
& <\frac{F_{4}(\beta)}{m^{2}(\beta)} .
\end{aligned}
$$

Hence the assertion (2.1) is proved. The proof of (2.2) is just the same as the proof of (2.1).

For the proof of Theorem 3 we first prove the following proposition.

Proposition 2. For any $\mu \in K_{j}$, any $\omega \in \Pi_{j}$ and every sufficiently large $\beta$, the following estimate is obtained,

$$
P_{V, \omega}\left(N_{\text {oil }}(\xi)=N\right)>c_{6}(\beta) \frac{1}{|V|^{\frac{1}{2}}},
$$

where $N$ is the positive integer satisfying $\left|N-n_{j}^{* *}\right| V||<F_{1}(\beta)|V|^{\frac{1}{2}}$ and $c_{6}(\beta)$ is the function of $\beta$ satisfying $c_{6}(\beta) \downarrow 0$ as $\beta \rightarrow \infty$.

The proof of this proposition is given in Appendix B. Taking into account the fact that the measure $P_{V, \omega}^{N}$ is independent of $\mu$, we have the following estimate for sufficiently large $V$,

$$
\begin{aligned}
& P_{V, \omega}^{N}\left(|| D(\xi)\left|-\delta^{*}\right| V||>g(\beta)|V|^{\frac{3}{4}+\alpha}\right) \\
&<\frac{P_{V, \omega}^{\mu}\left(|| D(\xi)\left|-\langle|D|\rangle_{V, \omega}\right|>\frac{1}{2} g(\beta)|V|^{\frac{3}{4}+\alpha}\right)}{P_{V, \omega}^{\mu}\left(N_{\text {oil }}(\xi)=N\right)},
\end{aligned}
$$

where $\mu \in K_{j}$. From Proposition 2 and Chebyshev's inequality, we have

$$
P_{V, \omega}^{N}\left(|| D(\xi)\left|-\delta^{*}\right| V \|>g(\beta)|V|^{\frac{3}{4}+\alpha}\right)<4 \frac{F_{4}(\beta)}{c_{6}(\beta) g^{2}(\beta)} \frac{1}{|V|^{2 \alpha}} .
$$

Hence, Theorem 3 is proved.

\section{Appendix A}

The purpose of this appendix is to prove Lemma 3.3 and Lemma 3.4. We state the proof in the following several steps. (See also [2].)

1) Let $V_{1}, V_{2}$ be two finite subsets of $\mathbf{Z}^{3}$, and $\partial\left(V_{1}, V_{2}\right)$ be the part of $\partial V_{1}$ which doesn't belong to $\partial V_{2}$.

$$
\begin{aligned}
& \chi_{V_{1}, \omega} \rho_{V_{1}, \omega}=\chi_{V_{1}, \omega} A \chi_{V_{1}, \omega} \rho_{V_{1}, \omega}+\chi_{V_{1}, \omega} \Lambda \\
& \chi_{V_{1}, \omega} \rho_{V_{2}, \omega}=\chi_{V_{1}, \omega} \chi_{V_{2}, \omega} \rho_{V_{2}, \omega}+\chi_{V_{1}, \omega} \Lambda .
\end{aligned}
$$

From (A.1) and (A.2), we have

where

$$
\eta_{V_{1}, V_{2}, \omega}=\chi_{V_{1}, \omega} A \eta_{V_{1}, V_{2}, \omega}+\zeta_{V_{1}, V_{2}, \omega}
$$

$$
\eta_{V_{1}, V_{2}, \omega}=\chi_{V_{1}, \omega} \rho_{V_{1}, \omega}-\chi_{V_{1}, \omega} \rho_{V_{2}, \omega}
$$


and

$$
\zeta_{V_{1}, V_{2}, \omega}=\chi_{V_{1}, \omega} A\left(\chi_{V_{1}, \omega} \rho_{V_{2}, \omega}-\chi_{V_{2}, \omega} \rho_{V_{2}, \omega}\right) \text {. }
$$

Let $\left\{\bar{B}_{1}, \ldots, \bar{B}_{s}\right\}$ be the set of $\omega$-type $B$-walls contained in $V_{1}$, then

$$
\begin{aligned}
\zeta_{V_{1}, V_{2}, \omega}\left(\bar{B}_{1}, \ldots, \bar{B}_{s}\right)= & \exp \left(-\beta E\left(\bar{B}_{1}\right)\right)\left[\sum_{k=1}^{\infty} \frac{(-1)^{k}}{k !} \sum_{\left\{\bar{F}_{i_{1}}, \ldots, \bar{F}_{l_{k}}\right\}} \rho_{V_{2}, \omega}\left(\bar{B}_{2}, \ldots, \bar{B}_{s}, \bar{F}_{i_{1}}, \ldots, \bar{F}_{i_{k}}\right)\right. \\
& \left.-\sum_{\bar{H}} \rho_{V_{2}, \omega}\left(\bar{B}_{2}, \ldots, \bar{B}_{s}, \bar{H}\right)\right],
\end{aligned}
$$

where the sum in the first terms extends over all $k$-pairs $\left\{\bar{F}_{i_{1}}, \ldots, \bar{F}_{i_{k}}\right\}$ each of which intersect or touch $B_{1}$ and at least one element of which intersect or surround $\partial\left(V_{1}, V_{2}\right)$, and the sum in the second term extends over all sets $\{\bar{H}\}$ which surround $B_{1}$ and intersect or surround $\partial\left(V_{1}, V_{2}\right)$. As in [2], we have

$$
\begin{aligned}
& \left|\zeta_{V_{1}, V_{2}, \omega}\left(\bar{B}_{1}, \ldots, \bar{B}_{s}\right)\right|
\end{aligned}
$$

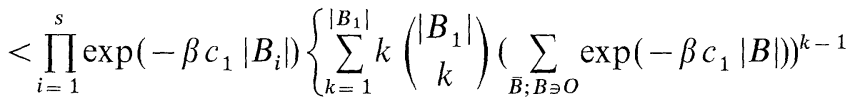

$$
\begin{aligned}
& \sum_{\bar{B} ; B \ni O,|B|>d\left(B_{1}, \partial\left(V_{1}, V_{2}\right)\right)} \exp \left(-\beta c_{1}|B|\right) \\
& \left.+\sum_{m=1}^{\infty} \sum_{\bar{B} ; B \ni O,|B|>m+d\left(B_{1}, \partial\left(V_{1}, V_{2}\right)\right)} \exp \left(-\beta c_{1}|B|\right)\right\} \\
& <c_{3}(\beta) \exp \left(-\left(\beta c_{1}-\ln c_{2}\right) d\left(B_{1}, \partial\left(V_{1}, V_{2}\right)\right)\right) \prod_{i=1}^{s} \exp \left(-\beta c_{1}\left|B_{i}\right|\right) \\
& \cdot\left\{\sum_{k=1}^{\left|B_{1}\right|} k\left(\begin{array}{c}
\left|B_{1}\right| \\
k
\end{array}\right) w(\beta)^{k-1}+\sum_{m=1}^{\infty} \exp \left(-\left(\beta c_{1}-\ln c_{2}\right) m\right)\right\} \\
& <D_{1}(\beta) \exp \left(-\left(\beta c_{1}-\ln c_{2}\right) d\left(B_{1}, \ldots, B_{s} ; \partial\left(V_{1}, V_{2}\right)\right) \prod_{i=1}^{s}\left(2 \exp \left(-\beta c_{1}\right)\right)^{\left|B_{2}\right|},\right.
\end{aligned}
$$

where

$$
D_{1}(\beta)=c_{3}(\beta)\left\{\sup _{k \geqq 8} k\left(\frac{1+w(\beta)}{2}\right)^{k-1}+\frac{\exp \left(-\left(\beta c_{1}-\ln c_{2}\right)\right)}{1-\exp \left(-\left(c_{1}-\ln c_{2}\right)\right)}\right\}
$$

2) For a given subset $W$ of $\mathbf{Z}^{3}$, we define the Banach space $\mathbf{X}(W)$ by

$$
\mathbf{X}(W)=\left\{\Phi \in \mathbf{X} ;\|\Phi\|_{W}<\infty\right\}
$$

where

$$
\begin{aligned}
\|\Phi\|_{W}= & \sup _{k \geqq 1}\left[\sup _{\left(\bar{B}_{1}, \ldots, \bar{B}_{k}\right)}\left|\phi_{k}\left(\bar{B}_{1}, \ldots, \bar{B}_{k}\right)\right| \prod_{i=1}^{k}\left(\frac{1}{2} \exp \left(\beta c_{1}\right)\right)^{\left|B_{i}\right|}\right. \\
& \left.\cdot \exp \left(\left(\beta c_{1}-\ln c_{2}\right) d\left(B_{1}, \ldots, B_{k}: W\right)\right)\right]
\end{aligned}
$$

The following lemma is proved similarly to the proof of Lemma 3.3 in [2]. 


\section{Lemma A.1.}

1) If $\Phi \in \mathbf{X}(W)$, then $A \Phi \in \mathbf{X}(W)$.

2) $\|A\|_{\mathbf{X}(W)}<1$ for sufficiently large $\beta$.

3) From (A.4), $\zeta_{V_{1}, V_{2}, \omega} \in \mathbf{X}\left(\partial\left(V_{1}, V_{2}\right)\right)$. From (A.3) and Lemma A.1 we have

$$
\begin{aligned}
& \left|\eta_{V_{1}, V_{2}, \omega}\left(\bar{B}_{1}, \ldots, \bar{B}_{s}\right)\right| \\
& \quad<\frac{D_{1}(\beta)}{1-D_{2}(\beta)} \exp \left(-\left(\beta c_{1}-\ln c_{2}\right) d\left(B_{1}, \ldots, B_{s}: \partial\left(V_{1}, V_{2}\right)\right) \prod_{i=1}^{s}\left(2 \exp \left(-\beta c_{1}\right)\right)^{\left|B_{i}\right|},\right.
\end{aligned}
$$

where $D_{2}(\beta)=\|A\|_{\mathbf{x}(W)}$.

By taking the limit as $V_{2} \rightarrow \mathbf{Z}^{3}$ and putting $V_{1}=V$, we obtain Lemma 3.3.

4) Taking into account the fact that

$$
\frac{\rho_{V, \omega}\left(\bar{B}_{1}, \bar{B}_{2}\right)}{\rho_{V, \omega}\left(\bar{B}_{1}\right)}=\rho_{V \backslash \theta\left(B_{1}\right), \omega}\left(\bar{B}_{2}\right),
$$

we obtain the following estimate from (A.3).

$$
\begin{aligned}
\mid \rho_{V, \omega} & \left(\bar{B}_{1}, \bar{B}_{2}\right)-\rho_{V, \omega}\left(\bar{B}_{1}\right) \rho_{V, \omega}\left(\bar{B}_{2}\right) \mid \\
& =\rho_{V, \omega}\left(\bar{B}_{1}\right)\left|\rho_{V \backslash \theta\left(B_{1}\right), \omega}\left(\bar{B}_{2}\right)-\rho_{V, \omega}\left(\bar{B}_{2}\right)\right| \\
& <c_{5}(\beta)\left(2 \exp \left(-c_{1} \beta\right)\right)^{\left|B_{1}\right|+\left|B_{2}\right|} \exp \left(-\left(\beta c_{1}-\ln c_{2}\right) d\left(B_{1}, B_{2}\right)\right),
\end{aligned}
$$

where $c_{5}(\beta)=\frac{D_{1}(\beta)}{1-D_{2}(\beta)}$.

\section{Appendix B}

In this appendix we prove Proposition 2 in the following several steps.

1) Let $N(\bar{\gamma})(\xi)$ be the number of outer $B$-walls which is congruent to $\bar{\gamma} \in \Gamma_{\omega}$ under the configuration $\xi \in \Omega_{V, \omega}$. As for the expectation value and variance of $N(\vec{\gamma})$, the following estimates are obtained by using Lemmas 3.2 and 3.3.

$$
\begin{gathered}
\langle N(\bar{\gamma})\rangle_{V \cdot \omega, \mu}=\sum_{\bar{B} \in \bar{\gamma}, B \subset V} \rho_{V, \omega}(\bar{B}) \quad\left(\omega \in \Pi_{j}, \mu \in K_{j}\right) \\
\langle N(\bar{\gamma})\rangle_{V, \omega}-\left.\rho(\bar{\gamma})|V|\left|<c_{7}(\beta)\right| \gamma\left|\exp \left(-\beta c_{1}|\gamma|\right)\right| V\right|^{\frac{1}{2}} \\
V_{V, \omega, \mu}(N(\bar{\gamma}))=\sum_{\substack{B_{1}, B_{2} \in \bar{\gamma} \\
B_{1}, B_{2} \subset V}}\left(\rho_{V, \omega, \mu}\left(\bar{B}_{1}, \bar{B}_{2}\right)-\rho_{V, \omega, \mu}\left(\bar{B}_{1}\right) \rho_{V, \omega, \mu}\left(\bar{B}_{2}\right)\right) \\
+\sum_{\substack{\bar{B} \in \bar{\gamma} \\
\bar{B} \subset V, \omega}} \rho_{V,(\bar{B})\left(1-\rho_{V, \omega}(\bar{B})\right),} \\
V_{V, \omega, \mu}(N(\bar{\gamma}))<c_{8}(\beta)|\gamma|^{2} \exp \left(-\beta c_{1}|\gamma|\right)|V| .
\end{gathered}
$$


2) Define

$$
\begin{aligned}
\mathbb{I} & =\left\{\xi \in \Omega_{V, \omega} ;|N(\bar{\gamma})(\xi)-\rho(\bar{\gamma})| V||\right. \\
& \left.\leqq B|\gamma| \exp \left(-\frac{1}{3} \beta c_{1}|\gamma|\right)|V|^{\frac{1}{2}} \text { for all } \bar{\gamma} \in \Gamma_{\omega}\right\} .
\end{aligned}
$$

By using Chebyshev's inequality, we obtain the following estimate.

$$
P_{V, \omega, \mu}(ף) \gg \frac{1}{3} \quad \text { for some } B>0 \text { and sufficiently large } \beta \text {. }
$$

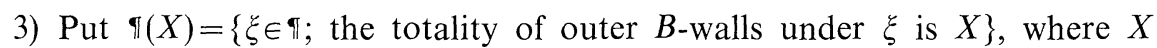
$=\left(\bar{B}_{1}, \ldots, \bar{B}_{s}\right)$ is a family of outer $B$-walls. For any $\omega \in \Pi_{j}$ and any $\mu \in K_{j}$, the expectation value of $N_{\text {oil }}$ in $\uparrow(X)$ is given by

$$
\left\langle N_{\text {oil }}\right\rangle_{q_{(X)}}=u_{j}|V|-\sum_{\bar{\gamma} \in \Gamma_{\omega}}\left(u_{j} v(\bar{\gamma})-\langle n(\bar{\gamma})\rangle\right) N(\bar{\gamma} ; X),
$$

where $N(\bar{\gamma} ; X)$ is the number of $B$-walls in $X$ which belong to $\bar{\gamma} \in \Gamma_{\omega}$. We note that

$$
\left|N(\bar{\gamma} ; X)-\rho_{\omega}(\bar{\gamma})\right| V||<B|\gamma| \exp \left(-\frac{1}{3} \beta c_{1}|\gamma|\right)|V|^{\frac{1}{2}}
$$

for all $\bar{\gamma} \in \Gamma_{\omega}$. Hence

$$
\begin{aligned}
\left|\left\langle N_{\mathrm{oi} 1}\right\rangle_{\mathbb{q}(X)}-\right| V \mid\left\{\sum_{\bar{\gamma} \in \Gamma_{\omega}}\left(\langle n(\bar{\gamma})\rangle-u_{j} v(\gamma) \rho(\bar{\gamma})+u_{j}\right\} \mid\right. \\
\quad<B|V|^{\frac{1}{2}} \sum_{\bar{\gamma} \in \Gamma_{\omega}}\left|\langle n(\bar{\gamma})\rangle-u_{j} v(\gamma)\right||\gamma| \exp \left(-\frac{1}{3} \beta c_{1}|\gamma|\right) \\
\quad \equiv \lambda(\beta)|V|^{\frac{1}{2}},
\end{aligned}
$$

where $(\beta) \downarrow 0$ exponentially as $\beta \rightarrow \infty$. Similarly we have

$$
V_{\mathbb{I}_{(X)}}\left(N_{\mathrm{oil}}\right)<\lambda(\beta)^{2}|V|
$$

4) We take one element $\bar{\gamma}_{0} \in \Gamma_{\omega}$ whose inner region $\operatorname{In}\left(\bar{\gamma}_{0}\right)$ is not vacant. Put

$$
\begin{aligned}
& M^{(1)}(\xi)=\sum_{\bar{B}_{i} \in X, \bar{B}_{\imath} \notin \bar{\gamma}_{0}}\left(v\left(\gamma_{0}\right)-N_{B_{\imath}}(\xi)\right) \\
& M^{(2)}(\xi)=\sum_{\bar{B}_{i} \in X, \bar{B}_{\imath} \notin \bar{\gamma}_{0}}\left(v\left(B_{i}\right)-N_{\bar{B}_{i}}(\xi)\right) \quad(\xi \in \mathbb{\Psi}(X)),
\end{aligned}
$$

where $N_{\bar{B}_{i}}(\xi)$ is the number of $o$-particles in $\theta\left(B_{i}\right)$. In the ensemble $₫(X)$ we can write

$$
N_{\text {oil }}(\xi)=|V|-M^{(1)}(\xi)-M^{(2)}(\xi)
$$

and $M^{(1)}(\xi)$ can be represented as a sum of independent identically distributed random variables numbering $N\left(\bar{\gamma}_{0} ; X\right)$ terms. As for the expectation value of 
$M^{(1)}$ in $₫(X)$, we have

$$
\left\langle M^{(1)}\right\rangle_{\pi_{(X)}}=\left(v\left(\bar{\gamma}_{0}\right)-\left\langle n\left(\bar{\gamma}_{0}\right)\right\rangle\right) N\left(\bar{\gamma}_{0} ; X\right) .
$$

Taking into account that

we have

$$
\left|N\left(\bar{\gamma}_{0} ; X\right)-\rho_{\omega}\left(\bar{\gamma}_{0}\right)\right| V||<B|\gamma| \exp \left(-\frac{1}{2} \beta c_{1}|\gamma|\right)|V|^{\frac{1}{2}}
$$

$$
D_{\Upsilon_{(X)}}\left(M^{(1)}\right)=N\left(\bar{\gamma}_{0} ; X\right) D\left(n\left(\bar{\gamma}_{0}\right)\right)>\frac{1}{2} \rho_{\omega}\left(\bar{\gamma}_{0}\right)|V|
$$

for sufficiently large $V$.

Because $N\left(\bar{\gamma}_{0} ; X\right)>\frac{1}{2} \rho\left(\bar{\gamma}_{0}\right)|V|$, we can apply the local limit theorem to $M^{(1)}$. Put $\kappa(\beta)=F_{1}(\beta)+3 \lambda(\beta)$.

For each $m$ satisfying $\left|m-\left\langle M^{(1)}\right\rangle_{\uparrow_{(X)}}\right|<\kappa(\beta)|V|^{\frac{1}{2}}$, we have

$$
\begin{aligned}
P_{\tau_{(X)}} & \left(M^{(1)}(\xi)=m\right) \\
& =\frac{1}{\sqrt{2 \pi N\left(\bar{\gamma}_{0} ; X\right) D\left(n\left(\bar{\gamma}_{0}\right)\right)}} \exp \left\{-\frac{\left(m-\left\langle M^{(1)}\right\rangle_{\uparrow(X)}\right)^{2}}{2 N\left(\bar{\gamma}_{0} ; X\right) D\left(n\left(\bar{\gamma}_{0}\right)\right)}\right\}\left(1+O\left(|V|^{-\frac{1}{2}}\right)\right) \\
& >\frac{1}{\sqrt{2 \pi \rho_{\omega}\left(\bar{\gamma}_{0}\right) D\left(n\left(\bar{\gamma}_{0}\right)\right)}|V|^{\frac{1}{2}}} \exp \left(-\frac{\kappa(\beta)^{2}}{\rho_{\omega}\left(\bar{\gamma}_{0}\right) D\left(n\left(\bar{\gamma}_{0}\right)\right)}\right)\left(1+O\left(|V|^{-\frac{1}{2}}\right)\right) \\
& >D(\beta)|V|^{-\frac{1}{2}},
\end{aligned}
$$

where $D(\beta)$ is some function of $\beta$.

From (B.7), (B.8) and (B.13), we have

$$
\begin{aligned}
& P_{\Upsilon_{(X)}}\left(N_{\text {oil }}(\xi)=N\right) \\
& =P_{\Upsilon_{(X)}}\left(M^{(1)}(\xi)+M^{(2)}(\xi)=|V|-N\right) \\
& >\sum_{\mid m-\left\langle M^{(1)}\right\rangle \uparrow_{\left.(X)|<\kappa(\beta)| V\right|^{\frac{1}{2}}}} P_{\Upsilon_{(X)}}\left(M^{(1)}(\xi)=m\right) P_{\Psi_{(X)}}\left(M^{(2)}(\xi)=|V|-N-m\right) \\
& >\frac{D(\beta)}{|V|^{\frac{1}{2}}} P_{\Psi_{(X)}}\left(\left|\left\langle N_{\text {oil }}\right\rangle_{\Psi_{(X)}}+\left\langle M^{(2)}\right\rangle_{\Psi_{(X)}}-N-M^{(2)}(\xi)\right|<\kappa(\beta)|V|^{\frac{1}{2}}\right) \\
& >\frac{D(\beta)}{|V|^{\frac{1}{2}}} P_{\mathbb{T}(X)}\left(\left|M^{(2)}(\xi)-\left\langle M^{(2)}\right\rangle_{\mathbb{\Psi}(X)}\right|<2 \lambda(\beta)|V|^{\frac{1}{2}}\right) \\
& >\frac{3}{4} \frac{D(\beta)}{|V|^{\frac{1}{2}}}
\end{aligned}
$$

Hence, we have

$$
\begin{aligned}
P_{V, \omega}\left(N_{\mathrm{oi} 1}(\xi)=N\right) & >P_{V, \omega}(\mathbb{\Uparrow}) P_{\uparrow}\left(N_{\mathrm{oil}}=N\right) \\
& >\frac{3}{8} \frac{D(\beta)}{|V|^{\frac{1}{2}}} .
\end{aligned}
$$

Acknowledgements. We thank Prof. Tokuzo Shiga and Prof. Yoichiro Takahashi for many valuable discussions and suggestions. We also thank Prof. Yuji Itho for reading the manuscript. 


\section{References}

1. Minlos, R.A., Sinai, Ja.G.: Mat. Sb. 73, 115 (1967)

2. Minlos, R.A., Sinai, Ja.G.: Trans. Moscow Math. Soc. 19, 121 (1968)

3. Heilmann, O.J.: Commun. Math. Phys. 36, 91 (1974)

4. Gertsik, V.M., Dobrushin, R.L.: Funkts. Anal. 8, 12 (1972)

5. Tachibana, T., Kobayashi, K., Suzuki, H., Honda, K., Sukigara, M.: Liquid crystal. Tokyo: Kyoritsu 1972 (in Japanese)

6. Peierls, R.E.: Proc. Cambridge Philos. Soc. 32, 477 (1936)

7. Dobrushin, R.L.: Funct. Anal. Appl. 8, 302 (1968)

8. Abraham, D.B., Heilmann, O.J.: J. Stat. Phys. 4, 15 (1972)

9. Abraham, D.B., Heilmann, O.J.: J. Stat. Phys. 13, 6, 461 (1975)

10. Gallavotti, G., Miracle-Sole, S.: Commun. Math. Phys. 27, 103 (1972)

11. Minlos, R.: Russ. Math. Surv. 23, 137 (1968)

12. Ruelle, D.: Statistical mechanics. Rigorous results. New York: Benjamin 1969

13. Miyamoto, M.: Phase transitions in lattice models. Seminar on Prob. (1972) (in Japanese)

Communicated by E. Lieb

Received January 21, 1981 